\title{
Identities for modular forms generated by vertex algebras
}

\author{
Alexander Zuevsky
}




\title{
IDENTITIES FOR MODULAR FORMS GENERATED BY VERTEX ALGEBRAS
}

\section{ALEXANDER ZUEVSKY}

\author{
Abstract. We review our recent results in the vertex algebra approach in construction of identities \\ for modular forms.
}

2000 Mathematics Subject Classification: 17B69, 30F10, 32A25, 11F03

Keywords: vertex operator superalgebras, intertwining operators, Riemann surfaces, modular forms, theta-functions, Frobenius-Fay and Jacobi product identities

\section{RECENT RESULTS}

The purpose of this review article is to describe our recent results as well as to announce results to appear towards understanding of the vertex operator origin of fundamental identities for modular forms. In particular, we review the results presented in the papers [6-11] accomplished in collaboration with M. P. Tuite (National University of Ireland, Galway).

Vertex algebras [1] give rise to non-trivial relations among the representation theory, analytic number theory, and algebraic geometry. In particular, they serve as an algebraic tool in generating modular forms as higher characters for vertex operator algebra modules. In the physics language, this corresponds to multiple-point correlation functions for appropriate conformal field theories defined on compact Riemann surfaces.

A complete description of all $n$-point functions for the Heisenberg bosonic and lattice VOAs in [4] and for fermionic vertex operator superalgebras in [5] has been given. Furthermore, employing the lattice bosonized version of these VOSAs leads to new Frobenius-Fay-type and Jacobi product identities for genus g half-integral theta series and Prime forms $[6-8,10,11]$. For further discussion of computation of vertex operator superalgebra $n$-point functions and their modular properties, see [12].

\subsection{The structure of twisted modules for vertex operator superagebras (VOSA)}

First, we have to describe suitable vertex operator modules and corresponding intertwining operators. The algebra of intertwining operators $y_{g}(v, z)$ for the Heisenberg vertex operator algebra extended by its modules generated by a family $g$ of continuous automorphisms is constructed explicitly. We introduced a $\mathbb{C}$-extension of 
the notion of a generalized vertex algebra [2] and proved that the intertwining operators constructed satisfy this definition. Explicit relations between shifted and twisted VOSA modules is also established [9].

\subsection{Geometry of reconstructing kernels on Riemann surfaces}

Reconstruction kernels (e. g. Cauchy kernel) play a very important role in computation of correlation functions for vertex operator modules. In particular, the Szegó kernel is suitable for the description of fermionic (half-integral graded) modules. Two alternative procedures of sewing (generalizing Yamada's construction) Riemann surfaces of arbitrary genuses are developed. We prove the modular properties and find explicit formulas for the Szegó kernel on a Riemann surface of genus $g_{1}+g_{2}$ (or $g+1$ ) obtained by using the corresponding kernels on two original genuses $g_{1}$ and $g_{2}$ Riemann surfaces [8].

\subsection{Computation of correlation functions in conformal field theory}

In a rigorous and constructive manner, we introduce the partition functions (graded dimensions) and correlations $n$-point functions (higher characters) for a twisted module $M_{f}$ of a vertex operator (super) algebra $V$ with the formal parameter associated to a complex parameter on a Riemann surface $\Sigma^{(g)}$ of genus $g \geq 1$ obtained by the surface surgery. A modification of the Zhu reduction procedure expressing a correlation $n+1$-point function via a finite sum of $n$-point functions with twisted higher Weierstrass functions as coefficients is introduced. In this algebraic approach, we can find closed formulas and explicitly establish modular properties of bosonic and fermionic characters on the torus, $q=e^{2 \pi i \tau}, z_{i} \in \Sigma^{(g)}, v_{i} \in V[5,6]$ :

$$
Z_{M_{f}}^{(1)}\left(v_{1}, z_{1}, \ldots, v_{n}, z_{n} ; q\right)=\operatorname{STr}_{M_{f}}\left(y_{f}\left(v_{1}, z_{1}\right) \ldots y_{f}\left(v_{n}, z_{n}\right) q^{L_{f}(0)-C / 24}\right),
$$

and at genus two $[7,10]$. Modularity properties of $n$-point functions with respect to appropriate group follows explicitly from the vertex operator derivation. The final expressions are given by the determinants of the matrices with elements being coefficients in the expansions of the regular parts of corresponding differentials (Bergman kernel $A_{a}, a=1,2$ for bosons, or Szegó kernel $Q$ for fermions) [5, 7, 8, 10]. Deep combinatorial properties of these formulas are established by means of the Principal MacMahon Theorem [6]. The 1-point function for the Virasoro vector satisfies the genus two Ward identity [8,9]. The partition functions on Riemann surfaces of arbitrary genuses formed in the Schottky uniformization were calculated [6].

\subsection{Twisted modular forms}

The theory of twisted elliptic quasi-modular forms with two real torsion characteristics associated with two parameters of the character over a vertex operator superalgebra twisted module is developed. Twisted Eisenstein series and higher Weierstrass 
functions are introduced and modularity with respect to the group $\operatorname{SL}(2, \mathbb{Z})$ is proved [5]. This theory can be generalized to the theory of twisted Jacobi forms.

\section{IDENTITIES FOR GENUS $g$ PARTITION FUNCTIONS}

In $[6,11]$, we consider the definition and computation of the genus $g$ partition function (in the multiple torus sewing and Schottky formations of a genus $g$ Riemann surface) for bosonic and fermionic vertex models.

\subsection{Genus $g$ partition function in sewing two Riemann surfaces}

We define [11] a genus $g$ partition function by inductively sewing together lower genus 1-point functions $Z_{M}^{\left(g_{i}\right)}\left(u, z_{i}\right), i=1,2, z_{i} \in \Sigma^{\left(g_{i}\right)}, u$ belongs to the $n$th grading subspace of $V$, for a $V$-module $M$ :

$$
Z_{M}^{(g)}(\epsilon)=\sum_{n \geq 0} \epsilon^{n} \sum_{u \in V_{[n]}} Z_{M}^{\left(g_{1}\right)}\left(u, z_{1}\right) Z_{M}^{\left(g_{2}\right)}\left(\bar{u}, z_{2}\right),
$$

where $g=g_{1}+g_{2}$ and $z_{1}, z_{2}$ are insertion points, $\epsilon$ is the sewing parameter, and $\bar{u}$, $u$ are related by a non-degenerate bilinear invariant form on $V$.

For instance, with suitable local coordinates in the neighbourhood of these points, we find for the Heisenberg VOA $M_{2}$, [11]

\section{Theorem 1.}

$$
Z_{M_{2}}^{(g)}(\epsilon)=\frac{1}{\operatorname{det}\left(I-Q_{\left(g_{1}, g_{2}\right)}\right)} Z_{M_{2}}^{\left(g_{1}\right)} Z_{M_{2}}^{\left(g_{2}\right)}
$$

where $Z_{M_{2}}^{(g)}(\epsilon)$ is a non-vanishing holomorphic function on the sewing domain and is automorphic with respect to $\mathrm{SL}(2, \mathbb{Z}) \times \ldots \times \mathrm{SL}(2, \mathbb{Z}) \subset \mathrm{Sp}(2 g, \mathbb{Z})$ with automorphy factor $\operatorname{det}\left(C \Omega^{(g)}+D\right)^{-1}$, and a multiplier system, $Q_{\left(g_{1}, g_{2}\right)}$ is a moment matrix containing genus $g$ sewing data, and $\Omega^{(g)}$ is a genus $g$ period matrix.

Consider the canonical embedding of $\gamma_{1}=\left(\begin{array}{ll}A_{1} & B_{1} \\ C_{1} & D_{1}\end{array}\right) \in \operatorname{Sp}\left(2 g_{1}, \mathbb{Z}\right)$,

$$
\gamma_{1} \hookrightarrow\left(\begin{array}{ll}
A & B \\
C & D
\end{array}\right)=\left(\begin{array}{cccc}
A_{1} & 0 & B_{1} & 0 \\
0 & I_{g_{2}} & 0 & 0 \\
C_{1} & 0 & D_{1} & 0 \\
0 & 0 & 0 & I_{g_{2}}
\end{array}\right) \in \mathrm{Sp}\left(2\left(g_{1}+g_{2}\right), \mathbb{Z}\right)
$$

Then, we prove the following identities [11]

\section{Theorem 2.}

$$
\operatorname{det}\left(I-\gamma_{1} \cdot Q_{\left(g_{1}, g_{2}\right)}\right)=\operatorname{det}\left(I-Q_{\left(g_{1}, g_{2}\right)}\right) \frac{\operatorname{det}\left(C \Omega^{\left(g_{1}+g_{2}\right)}+D\right)}{\operatorname{det}\left(C_{1} \Omega^{\left(g_{1}\right)}+D_{1}\right)},
$$

with $\gamma_{1} \cdot Q_{\left(g_{1}, g_{2}\right)}=Q_{\left(g_{1}, g_{2}\right)}\left(\gamma_{1} \cdot \Omega^{\left(g_{1}\right)}, \Omega^{\left(g_{2}\right)}\right)$. 


\subsection{Genus $g$ partition function for $g$ sewn tori}

One can find explicit formulas for various objects defined on the Riemann surface such as the bilinear form of the second kind, a basis of $g$ holomorphic 1 -forms and the genus $g$ period matrix in terms of either the Canonical or Schottky parametrizations [6].

We define the genus $g$ partition function for a VOSA $V$ in the canonical sewing scheme in terms of genus zero $2 g$-point correlation functions as follows:

$$
Z_{V}^{(g)}\left(\rho_{i}, A_{ \pm i}\right)=\left\langle\mathbf{1}, \prod_{i=1}^{g} \sum_{n_{i} \geq 0} \rho_{i}^{n_{i}} \sum_{v_{i} \in V^{(n)}} Y\left(v_{i}, A_{-i}\right) Y\left(\bar{v}_{i}, A_{i}\right) \mathbf{1}\right\rangle
$$

where $\mathbf{1}$ is the vacuum state in $V, \bar{v}_{i}$ is dual to $v_{i}$ with respect to a non-degenerate bilinear invariant form on $V$, and $\rho_{i}, i=1, \ldots, g A_{ \pm i}$ are the corresponding sewing parameters (see [6] for details).

In particular, the genus $g$ partition function can be computed for the rank 2 Heisenberg VOA by means of the MacMahon Master Theorem [10]. We then find for the Heisenberg VOA $M_{2}$ in canonical sewing of $g$ tori, [6]

\section{Theorem 3.}

$$
Z_{M_{2}}^{(g)}\left(\rho_{i}, A_{ \pm i}\right)=\frac{1}{\operatorname{det}\left(I-R^{\mathrm{Can}}\right)},
$$

where $R^{\text {Can }}$ is the canonical moment matrix. Furthermore, $\operatorname{det}\left(I-R^{\mathrm{Can}}\right)$ is a holomorphic non-vanishing function. In general, the genus $g$ Heisenberg generating function is expressed in terms of a permanent of genus $g$ bilinear forms of the second kind.

Alternatively, we may repeat this by using a definition of the genus $g$ partition function in terms of Schottky parameters which involve sums or products over the Schottky group [6]. We then find

Theorem 4. The genus $g$ partition function is

$$
Z_{M_{2}}^{(g)}\left(q_{i}, a_{ \pm i}\right)=\frac{1}{\operatorname{det}\left(I-R^{\mathrm{Sch}}\right)},
$$

where $R^{\text {Sch }}$ is the Schottky moment matrix, and $q_{i}, a_{ \pm i}$ are the corresponding sewing parameters. Furthermore, $\operatorname{det}\left(I-R^{\text {Sch }}\right)$ is a holomorphic non-vanishing function, and the genus $g$ Heisenberg generating function is expressed in terms of a permanent of genus $g$ bilinear forms of the second kind.

Then, it is natural to formulate the following

Conjecture. $\operatorname{det}\left(I-R^{\mathrm{Can}}\right)=\operatorname{det}\left(I-R^{\mathrm{Sch}}\right)$. 


\subsection{The Montonen-Zograf product formula}

The determinant $\operatorname{det}\left(I-R^{\mathrm{Sch}}\right)$ can also be re-expressed in terms of an infinite product formula originally calculated by Montonen. A similar product formula was subsequently found by Zograf. This has been recently related by McIntyre and Takhtajan to Mumford's theorem concerning the absence of a global section on moduli space for the canonical line bundle. Expressing the corresponding moments in terms of an $\operatorname{SL}(2, \mathbb{C})$ representation, we derive in [6] the identity

$$
\operatorname{det}\left(I-R^{\mathrm{Sch}}\right)=\prod_{m \geq 1} \prod_{\gamma^{\alpha} \in \Gamma}\left(1-q_{\alpha}^{m}\right)^{2},
$$

where the inner product ranges over the primitive classes $\gamma^{\alpha} \neq 1$ of the Schottky group $\Gamma$, i. e. $\gamma^{\alpha} \neq \gamma^{k}$ for any $\gamma \in \Gamma$ for $k \neq 1$. Each such element has a multiplier $q_{\alpha}$ where $\gamma^{\alpha}=\operatorname{diag}\left(q_{\alpha}, 1\right)$.

\section{GENUS $g \geq 1$ GENERALIZATIONS OF CLASSICAL IDENTITIES}

Bosonization formulas for $n$-point functions provide us with new genus $g$ generalizations of the classical elliptic Frobenius-Fay Trisecant identities for modular forms [5] as well as of Jacobi product identity [7]. For instance, on the torus, we obtain

$$
\begin{aligned}
\operatorname{det}\left(\mathbf{M}_{\mathbf{D}}\right)= & \frac{\vartheta\left(\begin{array}{c}
-\beta+\frac{1}{2} \\
\alpha+\frac{1}{2}
\end{array}\right)\left(\sum_{i=1}^{r} m_{i} z_{i}-\sum_{j=1}^{s} n_{j} z_{j}^{\prime}, \tau\right)}{\vartheta\left(\begin{array}{c}
-\beta+\frac{1}{2} \\
\alpha+\frac{1}{2}
\end{array}\right)(0, \tau)} \\
& \cdot \frac{\prod_{1 \leq i<k \leq r} K^{(1)}\left(z_{i}-z_{k}, \tau\right)^{m_{i} m_{k}} \prod_{1 \leq j<l \leq s} K^{(1)}\left(z_{j}^{\prime}-z_{l}^{\prime}, \tau\right)^{n_{j} n_{l}}}{\prod_{1 \leq i \leq r, 1 \leq j \leq s} K^{(1)}\left(z_{i}-z_{j}^{\prime}, \tau\right)^{m_{i} n_{j}}}
\end{aligned}
$$

where the matrix $\mathbf{M}_{\mathbf{D}}$ contains information on vertex operator expansions in torus $n$ point functions, and this formula relates it to product of powers of prime forms $K^{(1)}$ and theta functions.

For the torus case, the boson-fermion correspondence results in the classical (torus case) Jacobi triple identity [3]. From the vertex operator superalgebra side, its essence lies in the Mason-Tuite formula [4] relating torus lattice $n$-point functions and products of modular forms. For genus $g \geq 2$, Prime form similar identities can be conjectured. Nevertheless, for $g \geq 2$, we could make use of the genus one identities to derive higher genus analogues of Jacobi product identities [7,10]. In particular, at genus two, we get an explicit analogue of the classical (torus case, [3]) Jacobi triple 
identity [7]:

$$
\frac{\Theta^{(2)}\left(\begin{array}{l}
a \\
b
\end{array}\right)(\Omega)}{\vartheta^{(1)}\left(\begin{array}{l}
a_{1} \\
b_{1}
\end{array}\right)\left(\tau_{1}\right) \vartheta^{(1)}\left(\begin{array}{l}
a_{2} \\
b_{2}
\end{array}\right)\left(\tau_{2}\right)}=\operatorname{det}\left(I-A_{1}^{(1)} A_{2}^{(1)}\right)^{1 / 2} \operatorname{det}(I-Q) .
$$

Here, $\vartheta^{(1)}\left(\begin{array}{l}a \\ b\end{array}\right)(\tau)$, and $\Theta^{(2)}\left(\begin{array}{c}a \\ b\end{array}\right)(\Omega)$ are genus one and genus two theta-functions correspondingly, and $\Omega$ is a period matrix. Similar formulas in self-sewing of a Riemann surface (in $\rho$-formalism) are given in [10]. Generalizations for this type of identities are available for genus $g$ [11].

\section{Perspectives}

The results obtained stimulate further interest in application of algebraic technology of vertex algebras to number theory. We work now on procedures described above for super Riemann surfaces, on the identities for modular forms, $\theta$-functions and primary forms (in particular, generalizations of Macdonald-Kac identities) at genuses $g \geq 2$, the partition and $n$-point correlation functions, generation of twisted Jacobi forms, relations with co-operads, cluster algebras and structures generalizing vertex algebras [12], and applications of the theory of vertex algebras to Langlands program.

\section{REFERENCES}

[1] R. E. Borcherds, "Vertex algebras, Kac-Moody algebras and the Monster," Proc. Nat. Acad. Sc., vol. 83, pp. 3068-3071, 1986.

[2] C. Dong and J. Lepowsky, Generalized vertex algebras and relative vertex operators, ser. Progress in Mathematics. Boston, MA: Birkhäuser, 1993, vol. 112.

[3] V. Kac, Vertex Operator Algebras for Beginners, ser. University Lecture Series. Providence: American Mathematical Soc., 1998, vol. 10.

[4] G. Mason and M. P. Tuite, "Free bosonic vertex operator algebras on genus two Riemann surfaces I," Commun. Math. Phys., vol. 300, pp. 673-713, 2010.

[5] G. Mason, M. P. Tuite, and A. Zuevsky, "Torus $n$-point functions for $\mathbb{R}$-graded vertex operator superalgebras and continuous Fermion orbifolds," Commun. Math. Phys., vol. 283, no. 2, pp. 305-342, 2008.

[6] M. P. Tuite and A. Zuevsky, "The bosonic vertex operator algebra on a genus $g$ Riemann surface," RIMS Kokyuroko, vol. 1756, pp. 81-93, 2011.

[7] M. P. Tuite and A. Zuevsky, "Genus two partition and correlation functions for Fermionic vertex operator superalgebras I," Commun. Math. Phys., vol. 306, no. 2, pp. 419-447, 2011.

[8] M. P. Tuite and A. Zuevsky, "The Szegö kernel on a sewn Riemann surface," Commun. Math. Phys., vol. 306, no. 3, pp. 617-645, 2011.

[9] M. P. Tuite and A. Zuevsky, "A generalized vertex operator algebra for Heisenberg intertwiners," J. Pure and Applied Alg., vol. 216, no. 6, pp. 1253-1492, 2012.

[10] M. P. Tuite and A. Zuevsky, "Genus two partition and correlation functions for Fermionic vertex operator superalgebras II," 2012, 2012, http://arxiv.org/abs/1308.2441, to appear in Commun. Math. Phys. 2013. 
[11] M. P. Tuite and A. Zuevsky, "The Heisenberg vertex operator algebra on a genus $g$ Riemann surface," 2012, to appear.

[12] A. Zuevsky, "Twisted correlation functions on self-sewn Riemann surfaces via generalized vertex algebra of intertwiners," 2012, submitted to Springer Volume on Conformal Field Theory, Automorphic Forms and Related Topics, Heidelberg, 2011, Preprint 12-63, Max-Planck-Institut für Mathematik, Bonn, http://www.mpim-bonn.mpg.de/preblob/5340.

Author's address

Alexander Zuevsky

Max-Planck-Institut für Mathematik, Vivatsgasse 7, 53111 Bonn, Germany

E-mail address: zuevsky@mpim-bonn.mpg.de 